\title{
Metal structures diagnosis by truncated cone indentation
}

\author{
Alexey Beskopylny ${ }^{1, *}$, Andrey Veremeenko $^{1}$, Batyr Yazyev $^{1}$ \\ ${ }^{1}$ Don State Technical University, pl. Gagarina, 1, Rostov-on-Don, 344010, Russia
}

\begin{abstract}
The method of the steel structures diagnosis is considered with nondestructive test by truncated cone indentation. Recently, great interest in the nondestructive evaluating of the steel mechanical properties at real structures is developed in many applied problems. Dynamic indentation method is one of the most effective because of compatibility and accuracy. For this purpose static and dynamic problem of axisymmetric elasticplastic truncated cone indentation is solved and the results are compared with finite element analysis and experimental data. The method of nondestructive evaluating of mechanical characteristics is suggested and devise of the realization of the method is tested at real structures.
\end{abstract}

\section{Introduction}

Evaluation of mechanical properties of metal structures for various purposes is one of the most important tasks at any stage of their design and operation. The most important mechanical properties are yield strength, tensile strength, elongation, Charpy impact strength and hardness. These characteristics are necessary to know at all stages of the life cycle structure $[1,2]$. Non-destructive testing methods are prioritized especially for existing structures [3]. Recent researches show the possibility of assessment of the parameters of creep of materials for subsequent calculations [4,5,6,7].

It is known that one of the several ways to evaluate the mechanical characteristics is the method of pressing indenters various forms. The choice of the shape of the indenter in this case is an important task [8]. Most common in modern practice are indenters in the form of a sphere $[9,10]$, cone $[11,12]$, pyramid, flat punch [13], but they have one significant drawback - the complexity of the registration of the beginning of plastic flow. Great practical interest is the study of the influence of basic background stresses in the existing structures, under load, by the methods of indentation.

To register the moment of the start of plastic flow is possible truncated cone indenter that in the initial stages of pressing behaves as an elastic flat stamp, while the deformation of the sample is composed only of the elastic component and only after exceeding the threshold yield stress, plastic deformation occurs. In the second stage, a truncated cone starts to behave like a regular cone. Evaluation based on indentation force versus displacement of the indenter shows a turning point in the transition from elastic to plastic deformation. That it is possible to register for the tests.

\footnotetext{
*Corresponding author: besk-an@ya.ru
} 
There are several studies showing indentation of dual [14] and multiple indenter tips [15]. Great interest is the use of indenters of complex shape, consisting of two or more connected profiles without coupling. Indentation is carried out in coated parts in a quasistatic condition. Thus, in the first case, unlike the Rockwell diamond indenter was used the indenter combining nonconjugated sphere and cone. In the second - several conical surfaces with different cone angles that intersect each other, wherein the taper angle increases with distance from the tip of the indenter in steps. Such tests may be used for various kinds of plastic materials, including bimetal for parts, parts with coatings. It should be noted that number of theoretical studies conducted in this area is sufficiently small; most of the works are empirical. In order to develop testing methods frustoconical metal indentation is desirable to develop a model of contact interaction of the indenter in the form of a truncated cone with elastoplastic half-space and to evaluate their use.

\section{Problem statement}

The problem of indentation of an elastic truncated cone in elastic-plastic half-space by force $P$ that changes with time (Figure 1) is considered. We consider the cylindrical coordinates system; z-axis is directed to coincide with the axis of the cone and facing down (inside the half-space). In the contact area $\Omega_{1}(\alpha)$ of truncated cone with a half-space the friction is neglected, outside the area of contact $\square_{2}(\square)$ stresses $\sigma_{z r}$ and $\sigma_{z z}$ equate to zero. Plastic deformation begins under the Tresca conditions, the material at the same time characterizes the plastic constant $\mathrm{k}=0.5 \delta_{\text {yield }}$, and indenter material undergoes only elastic deformation.

In $[5,6,7]$ approximate solution of elastic-plastic problems is considered by solving two problems: the elastic and rigid-plastic with the following assumptions:

1. The displacement $u$ of any point of interacting solids is the sum of the elastic $u_{e}$ and plastic $u_{p}$ components:

$$
u=u_{e}+u_{p}
$$

In the Equation 1 displacement $u_{e}$ doesn't depend on the plastic deformation magnitude and is determined by the contact pressure, and the movement $u_{p}$ is constant when material is unloaded.

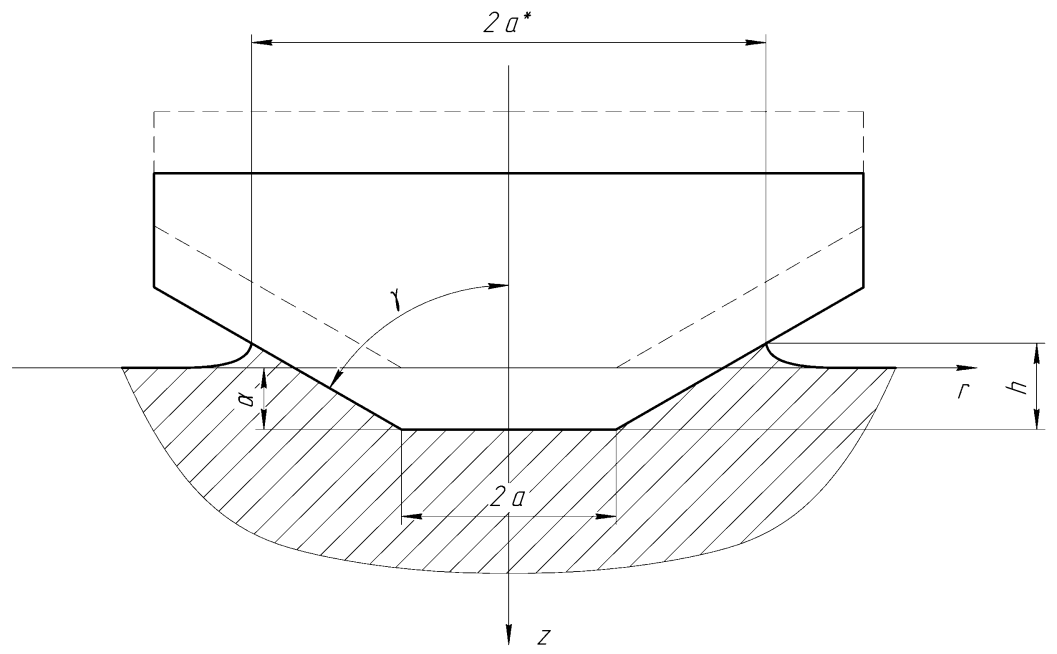

Fig.1. Diagram of indentation of a truncated cone. 
2. In the contact zone, the distribution of contact pressures is determined by the Equation 16 when $\square<\square_{c r}$

$$
q(P, r)=0.5 q_{0} \frac{a}{\sqrt{a^{2}-r^{2}}}, \quad q_{0}=P /\left(\pi a^{2}\right), \quad 0<r<a
$$

3. The average pressure in the contact zone does not exceed Brinell stresses (3).

$$
q_{0} \leq \lambda k, \quad \lambda \approx 5.7
$$

4. The growth of the plastic deformation occurs when the conditions (16) are performed

$$
q_{0}=\lambda k, \quad d P / d t>0
$$

The considered problem can be reduced to the problem of indentation of a stamp with a flat base in an elastic-plastic half space of the original flat surface. The half-space has elastic modulus $E^{*}$, defined by the Equation 16 and Poisson's ratio $v$ equal to zero. The stamp has a diameter equal to $2 a$ and the taper angle equal to $\gamma$ (Figure 1).

$$
E^{*}=E_{1} E_{2}\left[\left(1-v_{1}^{2}\right) E_{2}+\left(1-v_{2}^{2}\right) E_{1}\right]^{-1}
$$

At small value of force $P$, frustoconical indenter behaves as a flat stamp and according to [8] the elastic deformation and contact pressure can be determined from Equation 6 and 7.

$$
\begin{gathered}
\alpha=\frac{P}{2 E^{*} a} ; \\
q_{0}=\frac{2 \alpha E^{*}}{\pi a} .
\end{gathered}
$$

Plastic deformation takes place when the conditions (3) and (4) occur and when the critical values (8) and (9).

$$
\begin{aligned}
& \alpha_{c r}=\lambda k \pi \frac{a}{2 E^{*}}=\chi \frac{a}{2 E^{*}} ; \\
& P_{c r}=2 E^{*} a \alpha_{c r}=\chi a^{2},
\end{aligned}
$$

where $\chi=\lambda \mathrm{k} \pi$.

During the indentation (the indentation depth $\alpha$ is growth) plastic flow of material from under indenter occurs and in accordance with (2) and (4):

$$
a^{*}=(P / \chi)^{1 / 2}, \quad q=0.5 \lambda k \frac{a}{\sqrt{\left(a^{2}-r^{2}\right)}} .
$$

We consider a point located under the indenter and lying on the z-axis. According to research $[5,6,7,8,9]$, the plastic component of the depth of the hole is $h_{p}$ and the level of 
local indentation is $\alpha_{p}$ are connected by the Equation 11. The depth under elastoplastic deformation, as in Equation 1 is defined by the Equation 12.

$$
\alpha_{p}=\left(1-\delta^{*}\right) h_{p},
$$

$\delta^{*}-$ the value determined in the experiment.

$$
\alpha=\alpha_{p}+\alpha_{e}=\left(1-\delta^{*}\right)\left(h-h_{e}\right)+\alpha_{e}
$$

The depth $h$ (Figure 1) according to the geometry and elastic solutions $[7,8,9]$ can be defined by the Equation 13 .

$$
h=\operatorname{ctg}(\gamma)\left(a^{*}-a\right), \quad h_{e}=2 \alpha_{e} / \pi .
$$

Full indenter displasment:

$$
\begin{gathered}
\alpha=\left(1-\delta^{*}\right)\left(a^{*}-a\right) \operatorname{ctg}(\gamma)+\alpha_{e}(1-2 / \pi)= \\
=\left(1-\delta^{*}\right)\left(a^{*}-a\right) \operatorname{ctg}(\gamma)+\chi a^{*}(1-2 / \pi) /\left(2 E^{*}\right)= \\
=a^{*}\left[\left(1-\delta^{*}\right) \operatorname{ctg}(\gamma)+\chi(1-2 / \pi) /\left(2 E^{*}\right)\right]-\left(1-\delta^{*}\right) \operatorname{actg}(\gamma) .
\end{gathered}
$$

Given the condition Equation 4 and expression Equation 10 we obtain the depth at the elastic-plastic deformation

$$
\alpha=C(P / \chi)^{1 / 2}-C_{1}
$$

Parameter $C$ can be determined by:

$$
C=\left(1-\delta^{*}\right) \operatorname{ctg}(\gamma)+\chi(1-2 / \pi) /\left(2 E^{*}\right), \quad C_{1}=\left(1-\delta^{*}\right) \operatorname{actg}(\gamma)
$$

As a result, the dependence of $P(\alpha)$ can be written as a system of Equation 16 .

$$
\left\{\begin{array}{l}
P=2 a \alpha E^{*}, \quad \alpha \leq \alpha_{c r}, \\
P=\chi\left(\frac{\alpha+C_{1}}{C}\right)^{2}, \quad \alpha>\alpha_{c r} .
\end{array}\right.
$$

Thus, in the elastic deformation zone the linear relationship between the force and the indentation depth of penetration of half-space takes place, and this dependence becomes parabolic after reaching the critical load value. The turning point in the graph is clearly visible in the graphs $P(\alpha)$ when tested.

\section{Finite element analysis and experimental data}

To evaluate the stress-strain state of the material of half-space with the truncated cone indentation the widely known finite element method was applied. For modeling the elasticplastic properties of material, the flat four-node ISO-parametric elements were used. The study was carried out when the number of elements were changed from 250 to 800 , and nodes from 300 to 1500 .

For a more accurate solution to contact area mesh refinement was performed. It is known that during the static test a diamond or carbide indenter is applied, which has higher 
the rigidity than the rigidity of the elastic-plastic field. To simulate Tungsten alloy was used as more resistant than a diamond to the impact. Its mechanical properties are given by elastic modulus and Poisson's ratio.

For the simulation of the half space, the two models have been used in the plastic deformation zone that depends on the stress-strain: a bilinear isotropic hardening and multilinear kinematic hardening. As a result of solving the problem the stresses fields, displacement and strain were obtained, which allowed us to estimate characteristics of the deformation development from elastic to the elastic-plastic zone and to assess the increase in the contact area due to leakage of material from under the indenter.

Check the adequacy of the finite element models was carried out under static and dynamic loading. For experimental verification of the proposed models, static tests were conducted with the use of the press to determine the Rockwell hardness method. For the tests a number of indenters with taper angles $90^{\circ}$ and $120^{\circ}$ were made with a diameter of the flat part $2 a=0.42 \ldots 1.0 \mathrm{~mm}$. In addition, high-quality carbon and alloy steel were used. Steels properties are given in the table. During the tests, the values of the indenter force and the corresponding depth of his displacement was recorded on.

It should be noted that a small deviation from parallelism between the plane of the end face of the indenter and the plane of the sample, it is premature starts plastic deformation of the sample due to a high level stresses in the initial point of contact. To prevent this phenomenon the indenter rotates against the direction of rotation of the diamond-grinding wheel during the manufacture the end part of the truncated cone. The result of the clipping surface of the cone was a cone with an apex angle close to $2 \pi$. The analysis of the geometry of the imprint showed that the choice of this method of manufacture is successful, and the contact when the introduction occurs over the entire surface.

\section{Results and discussion}

A comparison of the results of an experimental study with the analytical model showed good agreement for steels with low strength (yield stress up to $700 \mathrm{MPa}$ ). When testing steels with higher strength, the importance of elastic deformation of the elements of the mechanism of the measuring device significantly increases, which complicates the registration process of moving the sample surface.

Graphics of the truncated cone indenter full displacement from the force of being pressed to several kinds of carbon steels is shown in Figure 2. It should be noted that the surface of real materials has a roughness that reduces the contact area therefore increases the magnitude of the contact pressure, the proportion of the elastic displacement leads to microplastic deformation. A significant influence on the error the effect of elastic component of deformation is occurred. There are high demands for the surface preparation to test for the accuracy of measuring instruments.

Figure 2 shows that the finite element method simulates the process of elastoplastic deformation very good and results are in good agreement with the experimental data. It is typical for many experimental conditions and different materials.

To check the adequacy of the model of impact interaction of indenter with the elasticplastic half-space the device was used in which the hitter is under spring tension hits with the indenter that is still standing on the sample. The indenter is mounted in the guide, in which is an inductive speed sensor. The signal from the sensor through the analog-to-digital Converter (ADC) goes to the computer for further processing. The sampling frequency of ADC up to $100 \mathrm{kHz}$, which allowed to conduct the poll signal with a time step of $10 \mu \mathrm{s}$. The indenter chosen for testing had a diameter of the planar part $2 a$ is equal to $0,42 \mathrm{~mm}$ and a cone angle $2 \gamma$ is equal to $120^{\circ}$. The result of the test was obtained according to kinematic parameters of the displacement $S(t)$, velocity $V(t)$ and acceleration $W(t)$ of time $t$. 
Table 1. The mechanical properties of the steels selected for static and impact tests.

\begin{tabular}{|c|c|c|c|c|}
\hline Steel & $\begin{array}{c}\text { Sample } \\
\text { number }\end{array}$ & $\begin{array}{c}\text { Yield stress, } \\
\text { MPa }\end{array}$ & $\begin{array}{c}\text { Tensile stress, } \\
\text { MPa }\end{array}$ & Elongation, \% \\
\hline 0,08 wt. \%C & 94 & 220 & 348 & 41.4 \\
\hline & 115 & 240 & 348 & 41.7 \\
\hline $0,14 \mathrm{C}, 2 \% \mathrm{Mn}$ & 1 & 281 & 491 & 33.3 \\
\hline & 2 & 282 & 490 & 32.0 \\
\hline & 3 & 276 & 488 & 33.0 \\
\hline & 4 & 281 & 489 & 32.7 \\
\hline $0,10 \% \mathrm{C}, 1 \% \mathrm{Cr}$, & 1 & 252 & 467 & 34.1 \\
\hline $1 \% \mathrm{Ni}, 1 \% \mathrm{Cu}$ & 2 & 255 & 476 & 32.9 \\
\hline & 2 & 409 & 618 & 24.4 \\
\hline & 3 & 408 & 615 & 22.3 \\
\hline & 4 & 407 & 611 & 24.4 \\
\hline $0,10 \% \mathrm{C}, 1 \% \mathrm{Cr}$, & 11 & 412 & 626 & - \\
\hline $1 \% \mathrm{Ni}, 1 \% \mathrm{Cu}$ & 332 & 536 & 32.3 \\
\hline & 12 & 340 & 544 & 31.4 \\
\hline & 14 & 345 & 552 & 29.6 \\
\hline
\end{tabular}

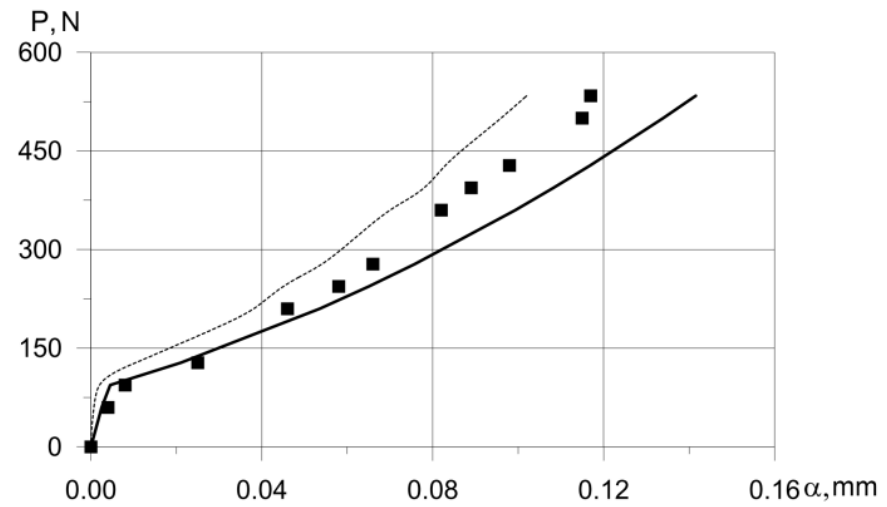

a)

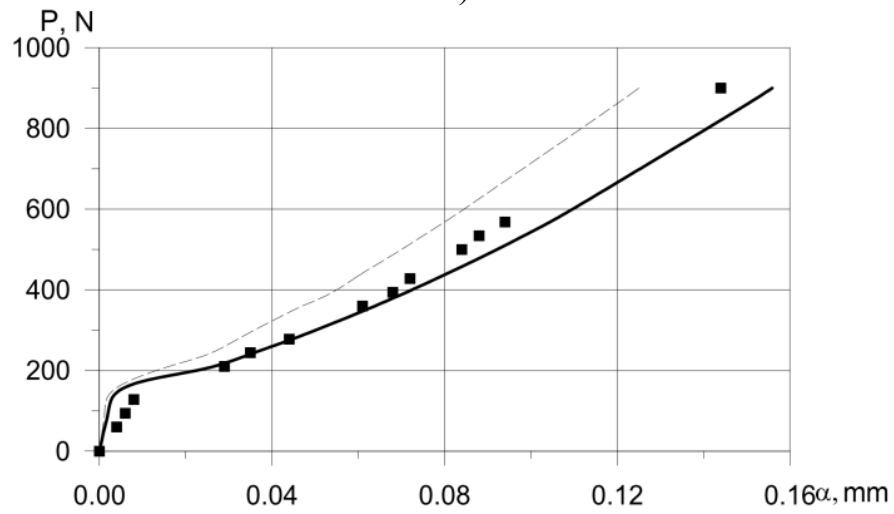

Fig. 2. The dependence of the displacement of the truncated cone on the indentation force $P$ for the (a) steel $0,08 \% \mathrm{C}$, (b) steel $0,10 \% \mathrm{C}, 1 \% \mathrm{Cr} \%, 1 \% \mathrm{Ni}, 1 \% \mathrm{Cu}$. 
Comparison of the results of theoretical and experimental studies have shown good convergence. Fig. 3 presents the dependence of the displacement $S(t)$, velocity $V(t)$ and the acceleration $W(t)$. The discrepancy between the results of theoretical and experimental studies in the determination of kinematic parameters was in the range: $6 \%$ for displacement, $10 \%$ for speed and $13 \%$ for acceleration.
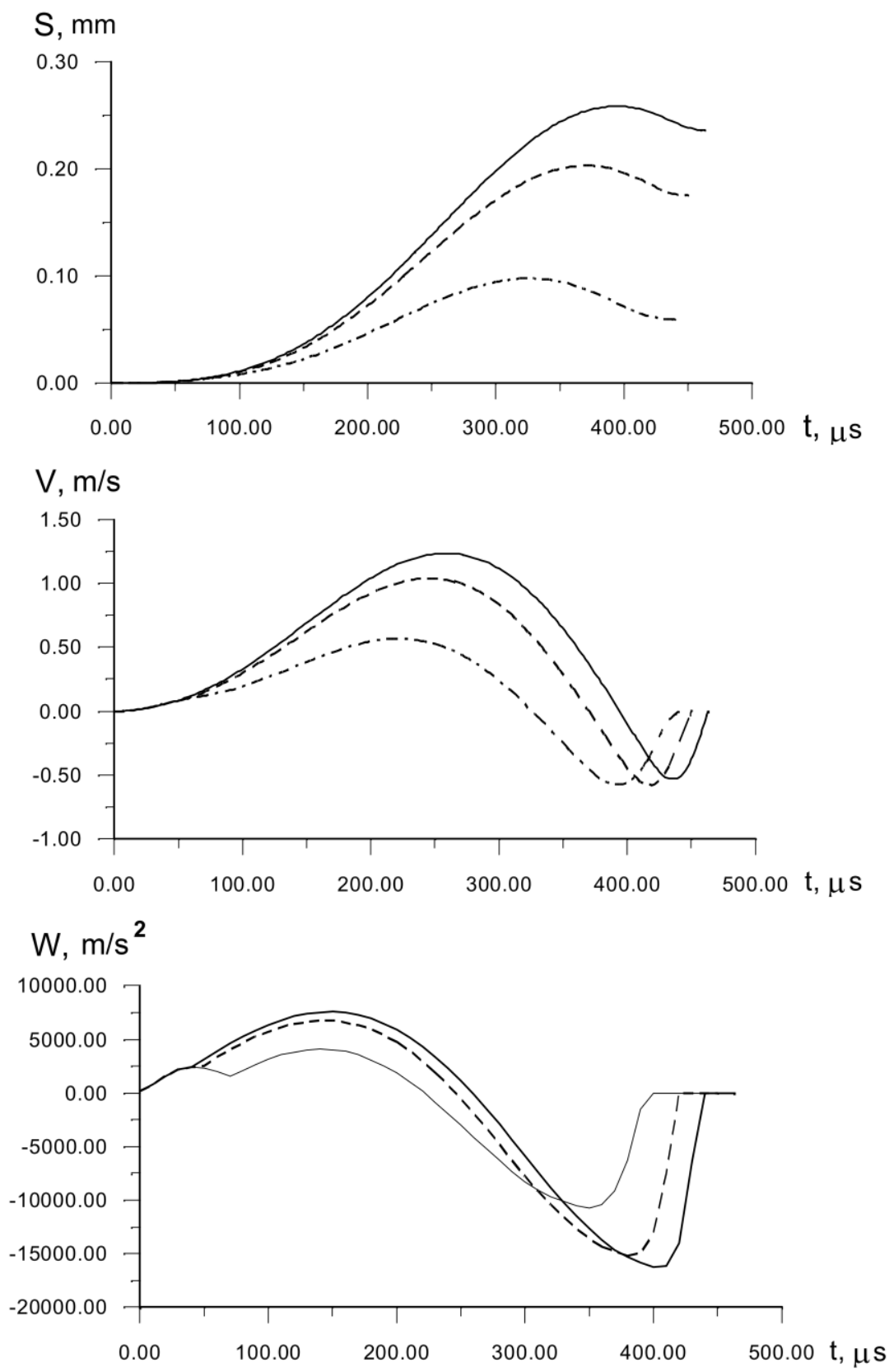

Fig. 3. The dependence of the displacement, velocity, and acceleration vs. time for a truncated cone at $a=0.21 \mathrm{~mm}$. 


\section{Conclusions}

The developed model showed that for ductile metals and alloys during the truncated cone indentation the peculiar turning point on the graph $P(\alpha)$ is observed that corresponding to the yield strength of the material. This phenomenon can be used to conduct both static and dynamic testing of these materials.

In this situation, it is appropriate to analyze the behavior of the models at various modes of interaction in order to establish the level of influence of various factors on the parameters of indentation. All this enables the development of methods of control of the main mechanical properties of materials on the kinematic parameters of truncated cone interaction with them. The truncated cone indentation in the early stages of interaction occurs elastic deformation. The duration of this phase is influenced by both external factors (indenter installation parameters, the plane part of the indenter, the impact energy and so on.), and the mechanical characteristics of the material - the modulus of elasticity and yield strength at first. Plastic flow begins after achieving critical indenter displacement $\alpha_{c r}$, in which material flows out of the stamp, forming a hole.

In the process of impact implementation in elastic contact the system of differential equations describing the movement of the indenter behaves linearly, resulting in the acceleration curve takes the form of a sine wave. In the event of plastic deformation reduced dependence is greatly disturbed that also leads to the fracture of the curve acceleration versus time.

Results of impact indentation of a truncated cone for several kinds of steels in the form of characteristic curves of displacement $S(t)$, the speed $V(t)$ of acceleration and $W(t)$ are shown in Fig. 3. This figure clearly visible, characterized turning point of transition from elastic deformation to plastic. Analysis of the behavior of graphs depending on the mechanical characteristics of the materials shows that the most significantly affected by the yield stress.

\section{References}

1. D.M. Belenkyi, A.N. Beskopylnyi, N.L. Vernesi, News of higher educational institutions. Construction 1, 99-102 (2003)

2. A.N. Beskopylny, I.G. Kadomtsev, N.I. Beskopylnaya, Theoretical foundations of civil engineering, 83-84 (2005)

3. A.N. Beskopylnyi, A.A. Lyapin, Recent trend in Science and Technology management 23-29, 36-45 (2016)

4. A.S. Chepurnenko, V.I. Andreev, B.M. Yazyev, A.N. Beskopylny, MATEC Web of Conferences 67, 06059 (2016)

5. V.I. Andreev, A.S. Chepurnenko, B.M. Yazyev, Advanced Materials Research 10041005, 257-260 (2014)

6. J. Dean, J. Campbell, G. Aldrich-Smith, T.W. Clyne, Acta Materialia 80, 56-66 (2014)

7. A.S. Vasiliev, S.S. Volkov, A.A. Belov, S.Yu. Litvinchuk, S.M. Aizikovich, International Journal of Engineering Science 112, 63-75 (2017)

8. A.R.H. Midawi, C.H.M. Simha, M.A. Gesing, A.P. Gerlich, International Journal of Solids and Structures 104-105, 81-91 (2017)

9. J. Dean, T.W. Clyne, Mechanics of Materials 105, 112-122 (2017)

10. A.S. Useinov, K.S. Kravchuk, A.A. Rusakov, I.V. Krasnogorov, A.P. Kuznetsov, T.V. Kazieva, Physics Procedia 72, 194-198 (2015) 
11. I. Mohagheghian, W.J. Stronge, G.J. McShane, European Journal of Mechanics 61, 134-150 (2017)

12. I.I. Argatov, International Journal of Solids and Structures 48, 3444-3452 (2011)

13. Wei-Min Chen, Yang-Tse Cheng, Min Li, Materials Science and Engineering 527, 5613-5618 (2010)

14. C. Heinrich, A.M. Waas, A.S. Wineman, International Journal of Solids and Structures 46, 364-376 (2009)

15. Minh-Quy Le, International Journal of Solids and Structures 45, 2818-2835 (2008) 